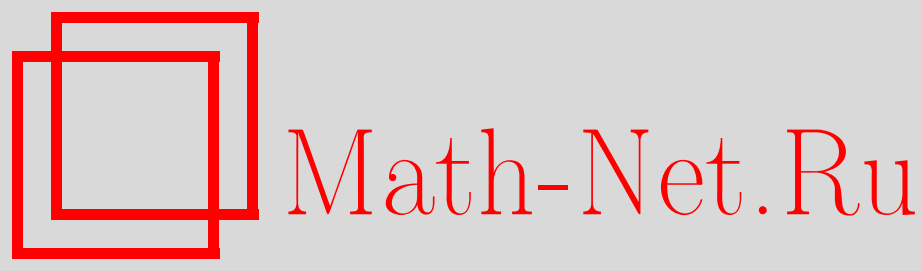

И. Я. Арефьева, Р. В. Горбачев, П. Б. Медведев, Вакуумные решения в полевой теории Невё-Шварца фермионной струны и представление нулевой кривизны в градуированных пространствах, ТМФ, 2009, том 159, номер 1, 131-141

DOI: https://doi.org/10.4213/tmf6337

Использование Общероссийского математического портала Math-Net.Ru подразумевает, что вы прочитали и согласны с пользовательским соглашением http://www . mathnet.ru/rus/agreement

Параметры загрузки:

IP : 3.80 .253 .173

26 апреля 2023 г., 15:51:54

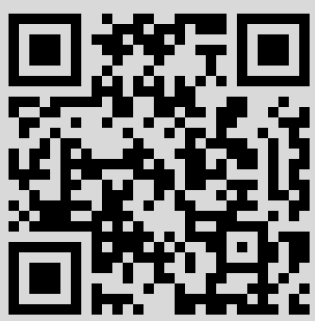




\section{ВАКУУМНЫЕ РЕШЕНИЯ В ПОЛЕВОЙ ТЕОРИИ НЕВЁ-ШВАРЦА ФЕРМИОННОЙ СТРУНЫ И ПРЕДСТАВЛЕНИЕ НУЛЕВОЙ КРИВИЗНЫ В ГРАДУИРОВАННЫХ ПРОСТРАНСТВАХ}

Показано, что вакуумные решения в полевой теории Невё-Шварца фермионной струны, включающие GSO(-)-сектор, естественным образом связаны с представлением нулевой кривизны в градуированном пространстве специального вида. Это же представление использовано для описания эквивалентности кубической и неполиномиальных теорий также с учетом $\operatorname{GSO}(-)$-сектора.

Ключевые слова: струнная теория поля, представление нулевой кривизны, чисто калибровочное решение.

\section{1. ВВЕДЕНИЕ}

Ковариантная полевая теория струны Невё-Шварца-Рамона (NSR-струны) в секторе Невё-Шварца (NS-секторе), преодолевающая трудности первоначального подхода Виттена [1] и имеющая действие типа Черна-Саймонса [2], [3], имеет в качестве уравнения движения в $\mathrm{GSO}(+)-\mathrm{NS}-$ секторе уравнение нулевой кривизны

$$
Q \Psi+\Psi^{2}=0
$$

Здесь $\Psi$ - элемент струнной алгебры, снабженной умножением $\star$ и оператором дифференцирования $Q$, которые удовлетворяют естественным свойствам, в частности правилу Лейбница

$$
Q\left(\Psi_{1} \star \Psi_{2}\right)=Q \Psi_{1} \star \Psi_{2}+(-1)^{\left|\Psi_{1}\right|} \Psi_{1} \star Q \Psi_{2},
$$

где $|\Psi|$ - четность элемента $\Psi$ (см. обзор [4]). Представление нулевой кривизны

$$
\Psi=\Omega^{-1} Q \Omega
$$

* Математический институт им. В. А. Стеклова РАН, Москва, Россия.

E-mail: arefeva@mi.ras.ru,rgorbachev@yandex.ru

${ }^{\dagger}$ Институт теоретической и экспериментальной физики, Москва, Россия 
где $\Omega$ - четный элемент струнной алгебры, является решением уравнения (1). Это утверждение основано на равенстве

$$
Q \Omega^{-1}=-\Omega^{-1}(Q \Omega) \Omega^{-1}
$$

Формула (2) является основой для построения вакуумных решений, описывающих конденсацию тахиона бозонной струны, также имеющей действие типа Черна-Саймонса. Само решение, описывающее тахионную конденсацию бозонной струны, отличается на так называемую аномальную часть от решения, которое имеет структуру чистой калибровки. Причем именно аномальная часть этого решения вносит существенный вклад в действие [5], [6] (см. также обсуждение и развитие работы Шнабла в статьях [7]).

Тахионная конденсация в NSR-струне описывается с помощью подключения GSO(-)-сектора, и уравнения движения в этой теории имеют вид [8]

$$
\begin{aligned}
& Q \Phi_{+}+\Phi_{+} \star \Phi_{+}-\Phi_{-} \star \Phi_{-}=0, \\
& Q \Phi_{-}+\Phi_{+} \star \Phi_{-}-\Phi_{-} \star \Phi_{+}=0 .
\end{aligned}
$$

Представление, являющееся аналогом условия нулевой кривизны для уравнения (4) построено в работе [9], и, на первый взгляд, оно не обладает структурой представления (2) при $\Phi_{-} \neq 0$.

Действие в теории, приводящей к уравнениям (4), может быть представлено как действие типа Черна-Саймонса [10] за счет перехода к матричнозначному полю

$$
\widehat{\Phi}=\Phi_{+} \otimes \sigma_{3}+\Phi_{-} \otimes i \sigma_{2}
$$

и матричнозначному BRST-заряду

$$
\widehat{Q}=Q \otimes \sigma_{3},
$$

где $\sigma_{i}$ - матрица Паули, $i=1,2,3$. При этом мы предполагаем, что грассмановы четности полей $\Phi_{+}$и $\Phi_{-}$противоположны. Если определить четность матричного поля (5) как $|\widehat{\Phi}| \equiv\left|\Phi_{+}\right|$, то справедливо правило Лейбница для матричнозначного BRST-заряда:

$$
\widehat{Q}(\widehat{\Phi} \star \widehat{\Psi})=(\widehat{Q} \widehat{\Phi}) \star \widehat{\Psi}+(-1)^{|\widehat{\Phi}|} \widehat{\Phi} \star(\widehat{Q} \widehat{\Psi}) .
$$

В матричных обозначениях система (4) записывается как уравнение

$$
\widehat{Q} \widehat{\Phi}+\widehat{\Phi} \star \widehat{\Phi}=0
$$

Естественно считать, что это уравнение имеет решение вида

$$
\widehat{\Phi}=\widehat{\Omega}^{-1} \widehat{Q} \widehat{\Omega}
$$

и это действительно так, если $\widehat{\Omega}$ обладает определенной четностью и справедлив аналог формулы (3). Однако если интересоваться решением, в котором $\widehat{\Omega}$ не имеет определенной четности (т.е. матричные элементы $\widehat{\Omega}$ имеют различную четность), то 
формула (9), вообще говоря, не описывает решение уравнения (8). Тем не менее, сделав дополнительные предположения о структуре $\widehat{\Omega}$, мы можем утверждать, что формула (9) дает решение уравнения (8). Вывод такой формулы и связь ее с решением, полученным в работе [9], и являются основными результатами настоящей работы. Мы также используем эту формулу для установления связи кубической теории, включающей GSO(-)-сектор [8], с соответствующей неполиномиальной теорией [11].

Решение, описывающее тахионную конденсацию в полной (т.е. включающей GSO(-)-сектор) струне, отличается на аномальную часть от специальных матричных представлений нулевой кривизны, которые связаны с представлением, построенным в работе [9].

Статья организована следующим образом. Раздел 2 содержит вывод представления нулевой кривизны в матричной теории, раздел 3 посвящен построению решения уравнения (8) методом итераций, показано, что решение, полученное методом итераций для матричного уравнения, воспроизводит решение, полученное методом итераций для системы (4). В разделе 4 устанавливается эквивалентность классических решений в теории (8) и неполиномиальной теории в матричной форме.

\section{2. ДЕЙСТВИЕ И УРАВНЕНИЕ В МАТРИЧНЫХ ОБОЗНАЧЕНИЯХ}

Струнно-полевое действие для NS-сектора с учетом GSO(-)-сектора, единственным образом обобщающее действие для NS-струны в GSO(+)-секторе [2], [3], имеет вид [8]

$$
\begin{aligned}
S\left[\Phi_{+}, \Phi_{-}\right]= & -\frac{1}{g_{0}^{2}}\left[\frac{1}{2}\left\langle Y_{-2} \Phi_{+}, Q \Phi_{+}\right\rangle+\frac{1}{3}\left\langle Y_{-2} \Phi_{+}, \Phi_{+}, \Phi_{+}\right\rangle+\right. \\
& \left.+\frac{1}{2}\left\langle Y_{-2} \Phi_{-}, Q \Phi_{-}\right\rangle-\left\langle Y_{-2} \Phi_{+}, \Phi_{-}, \Phi_{-}\right\rangle\right] .
\end{aligned}
$$

Здесь множители перед скобками с нечетным числом полей фиксированы условием калибровочной инвариантности действия. Для дальнейшего важно, что $\Phi_{+}-$ нечетное поле, а $\Phi_{-}$- четное поле. Варьируя действие по полям $\Phi_{+}, \Phi_{-}$, получаем уравнения движения (4) (с точностью до ядра оператора $Y_{-2}$ ). Действие (10) в матричных обозначениях (5) может быть записано как

$$
S[\widehat{\Phi}]=-\frac{1}{g_{0}^{2}}\left[\frac{1}{2}\left\langle\widehat{Y}_{-2} \widehat{\Phi}, \widehat{Q} \widehat{\Phi}\right\rangle+\frac{1}{3}\langle\widehat{\Phi}, \widehat{\Phi}, \widehat{\Phi}\rangle\right],
$$

где $\widehat{Y}_{-2}=Y_{-2} \otimes \sigma_{3}$, при этом уравнения движения принимают вид (9). Заметим, что действие $S[\widehat{\Phi}]$ инвариантно относительно инфинитезимальных преобразований $\delta \widehat{\Phi}=\widehat{Q} \widehat{\Lambda}+[\widehat{\Phi}, \widehat{\Lambda}]$, где $\Lambda-$ калибровочное поле. Здесь и в дальнейшем умножение

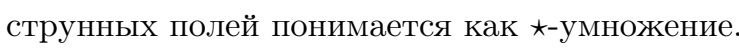

Непосредственное вычисление показывает, что из уравнения (8) с учетом (6) и (5) следуют уравнения (4). Напомним, что поля $\Phi_{+}$и $\Phi_{-}$являются полями различной четности. Именно поэтому представление нулевой кривизны мы запишем в виде

$$
\widehat{\Phi}=e^{-\Lambda_{+} \otimes I} e^{-\Lambda_{-} \otimes \sigma_{1}} \widehat{Q} e^{\Lambda_{-} \otimes \sigma_{1}} e^{\Lambda_{+} \otimes I} .
$$


Такая запись позволяет контролировать различные четности полей $\Lambda_{+}$и $\Lambda_{-}\left(\Lambda_{+}-\right.$ четное поле, $\Lambda_{-}-$нечетное поле).

На поле $\widehat{\Phi}$ следует наложить дополнительные условия $\operatorname{Tr} \widehat{\Phi}=0$ и $\operatorname{Tr}\left(\widehat{\Phi} \sigma_{1}\right)=0$. При этом оказывается, что представление нулевой кривизны (12) приводит к следующим представлениям для суммы и разности полей $\Phi_{+}$и $\Phi_{-}$:

$$
\begin{aligned}
& \Phi_{+}+\Phi_{-}=e^{-\Lambda_{+}} e^{\Lambda_{-}} Q\left(e^{\Lambda_{-}} e^{\Lambda_{+}}\right), \\
& \Phi_{+}-\Phi_{-}=e^{-\Lambda_{+}} e^{-\Lambda_{-}} Q\left(e^{-\Lambda_{-}} e^{\Lambda_{+}}\right) .
\end{aligned}
$$

Заметим, что эти соотношения не являются представлениями нулевой кривизны, так как знаки у $\Lambda_{-}$"неправильные". В конце данного раздела мы приведем эти формулы к более поучительному виду.

Формулы (13) следуют из уравнения (12) в силу соотношений

$$
e^{\Lambda_{-} \otimes \sigma_{1}} e^{\Lambda_{+} \otimes I}=\operatorname{ch} \Lambda_{-} e^{\Lambda_{+}} \otimes I+\operatorname{sh} \Lambda_{-} e^{\Lambda_{+}} \otimes \sigma_{1}=\Lambda_{1} \otimes I+\Lambda_{2} \otimes \sigma_{1} .
$$

Действительно,

$$
\begin{aligned}
e^{-\Lambda_{+} \otimes I} & e^{-\Lambda_{-} \otimes \sigma_{1}} Q \otimes \sigma_{3} e^{\Lambda_{-} \otimes \sigma_{1}} e^{\Lambda_{+} \otimes I}= \\
= & e^{-\Lambda_{+}}\left(\operatorname{ch} \Lambda_{-} \otimes I-\operatorname{sh} \Lambda_{-} \otimes \sigma_{1}\right) Q \otimes \sigma_{3}\left(\operatorname{ch} \Lambda_{-} \otimes I+\operatorname{sh} \Lambda_{-} \otimes \sigma_{1}\right) e^{\Lambda_{+}}= \\
= & e^{-\Lambda_{+}}\left[\operatorname{ch} \Lambda_{-} \cdot Q\left(\operatorname{ch} \Lambda_{-} e^{\Lambda_{+}}\right)+\operatorname{sh} \Lambda_{-} \cdot Q\left(\operatorname{sh} \Lambda_{-} e^{\Lambda_{+}}\right)\right] \otimes \sigma_{3}+ \\
& +e^{-\Lambda_{+}}\left[\operatorname{ch} \Lambda_{-} \cdot Q\left(\operatorname{sh} \Lambda_{-} e^{\Lambda_{+}}\right)+\operatorname{sh} \Lambda_{-} \cdot Q\left(\operatorname{ch} \Lambda_{-} e^{\Lambda_{+}}\right)\right] \otimes i \sigma_{2} .
\end{aligned}
$$

Следовательно,

$$
\begin{aligned}
& \Phi_{+}=e^{-\Lambda_{+}}\left[\operatorname{ch} \Lambda_{-} \cdot Q\left(\operatorname{ch} \Lambda_{-} e^{\Lambda_{+}}\right)+\operatorname{sh} \Lambda_{-} \cdot Q\left(\operatorname{sh} \Lambda_{-} e^{\Lambda_{+}}\right)\right] \\
& \Phi_{-}=e^{-\Lambda_{+}}\left[\operatorname{ch} \Lambda_{-} \cdot Q\left(\operatorname{sh} \Lambda_{-} e^{\Lambda_{+}}\right)+\operatorname{sh} \Lambda_{-} \cdot Q\left(\operatorname{ch} \Lambda_{-} e^{\Lambda_{+}}\right)\right]
\end{aligned}
$$

откуда непосредственно вытекают соотношения (13).

Легко проверить, что $\Phi_{+}+\Phi_{-}$и $\Phi_{+}-\Phi_{-}$из (13) являются решениями системы уравнений

$$
\begin{aligned}
& Q\left(\Phi_{+}+\Phi_{-}\right)+\left(\Phi_{+}-\Phi_{-}\right)\left(\Phi_{+}+\Phi_{-}\right)=0, \\
& Q\left(\Phi_{+}-\Phi_{-}\right)+\left(\Phi_{+}+\Phi_{-}\right)\left(\Phi_{+}-\Phi_{-}\right)=0,
\end{aligned}
$$

которая эквивалентна исходной системе (4). Действительно, воспользуемся соотношением

$$
Q e^{-\Lambda}=-e^{\Lambda}\left(Q e^{\Lambda}\right) e^{-\Lambda}
$$

которое выполняется для нечетных элементов $\Lambda$, поскольку $Q(\Lambda b)=(Q \Lambda) b-\Lambda Q b$. Формула (17) следует из того, что

$$
\begin{aligned}
0 & =Q\left(e^{-X} e^{X}\right)=Q\left(\left(1-X+\frac{X^{2}}{2 !}+\cdots\right)\left(1+X+\frac{X^{2}}{2 !}+\cdots\right)\right)= \\
& =Q\left(e^{-X}\right) e^{X}+\left(1+X+\frac{X^{2}}{2 !}+\cdots\right) Q e^{X}=Q\left(e^{-X}\right) e^{X}+e^{X} Q e^{X} .
\end{aligned}
$$


Таким образом, формула (17) является аналогом формулы (3) для нечетных относительно действия $Q$ полей $\Lambda$.

Проверим явно, что из соотношений (13) с учетом (17) следует (15). Имеем

$$
\begin{aligned}
Q\left(\Phi_{+}+\Phi_{-}\right) & =Q\left(e^{-\Lambda_{+}} e^{\Lambda_{-}} Q\left(e^{\Lambda_{-}} e^{\Lambda_{+}}\right)\right)= \\
& =\left(Q e^{-\Lambda_{+}}\right) e^{\Lambda_{-}} Q\left(e^{\Lambda_{-}} e^{\Lambda_{+}}\right)+e^{-\Lambda_{+}}\left(Q e^{\Lambda_{-}}\right) Q\left(e^{\Lambda_{-}} e^{\Lambda_{+}}\right) .
\end{aligned}
$$

С другой стороны,

$$
\left(\Phi_{+}-\Phi_{-}\right)\left(\Phi_{+}+\Phi_{-}\right)=e^{-\Lambda_{+}} e^{-\Lambda_{-}} Q\left(e^{-\Lambda_{-}} e^{\Lambda_{+}}\right) e^{-\Lambda_{+}} e^{\Lambda_{-}} Q\left(e^{\Lambda_{-}} e^{\Lambda_{+}}\right) .
$$

Применяя формулу (17), а также учитывая, что (в силу тех же соображений, что и при выводе (17))

$$
Q\left(e^{-\Lambda_{-}} e^{\Lambda_{+}}\right)=\left(Q e^{-\Lambda_{-}}\right) e^{\Lambda_{+}}+e^{\Lambda_{-}} Q e^{\Lambda_{+}},
$$

получаем

$$
\left(\Phi_{+}-\Phi_{-}\right)\left(\Phi_{+}+\Phi_{-}\right)=-\left(Q e^{-\Lambda_{+}}\right) e^{\Lambda_{-}} Q\left(e^{\Lambda_{-}} e^{-\Lambda_{+}}\right)-e^{-\Lambda_{+}} Q e^{\Lambda_{-}} Q\left(e^{\Lambda_{-}} e^{\Lambda_{+}}\right),
$$

и это с точностью до знака дает правую часть равенства (19). Так же доказывается справедливость уравнения (16).

С помощью формулы (21) можно привести равенства (13) к виду

$$
\Phi_{+}+\Phi_{-}=e^{-\Lambda_{+}}\left(\chi_{+}+Q\right) e^{\Lambda_{+}}, \quad \Phi_{+}-\Phi_{-}=e^{-\Lambda_{+}}\left(\chi_{-}+Q\right) e^{\Lambda_{+}},
$$

где $\chi_{ \pm}=e^{ \pm \Lambda_{-}} Q e^{ \pm \Lambda_{-}}$, т.е. $\Phi_{+} \pm \Phi_{-}$получаются калибровочным преобразованием из полей $\chi_{ \pm}$.

\section{3. РЕШЕНИЕ МАТРИЧНОГО УРАВНЕНИЯ ДВИЖЕНИЯ МЕТОДОМ ИТЕРАЦИЙ}

В работе [9] решение уравнения движения (8) было найдено итерационным методом для каждой из компонент. В этом разделе мы покажем, как с помощью итерационного метода найти решение матричного уравнения (8).

Разложим $\hat{\phi}$ в ряд по параметру $\lambda$ :

$$
\hat{\phi}=\sum_{n=0}^{\infty} \lambda^{n+1} \hat{\phi}_{n} ;
$$

подставив это разложение в уравнение движения (8), в первом порядке по $\lambda$ имеем $\widehat{Q} \hat{\phi}_{0}=0$. Частное решение этого уравнения есть $\hat{\phi}_{0}=\widehat{Q} \hat{\phi}$, где $\phi_{+}$и $\phi_{-}$суть компоненты калибровочного поля $\hat{\phi}=\phi_{+} \otimes I+\phi_{-} \otimes \sigma_{1}$, принадлежащие соответственно $\operatorname{GSO}(+)$ - и $\operatorname{GSO}(-)$-секторам и имеющие противоположные грассмановые четности. Во втором порядке по $\lambda$ имеем $\widehat{Q} \hat{\phi}_{1}+\hat{\phi}_{0} \hat{\phi}_{0}=0$. Для $\hat{\phi}_{0}=\widehat{Q} \hat{\phi}$, учитывая правило Лейбница (7) для $\widehat{Q}$, получаем

$$
\widehat{Q} \hat{\phi}_{1}+\widehat{Q} \hat{\phi} \star \widehat{Q} \hat{\phi}=\widehat{Q}\left(\hat{\phi}_{1}-\widehat{Q} \hat{\phi} \star \hat{\phi}\right)=0 .
$$


Знак минус обусловлен тем, что мы выбираем $|\hat{\phi}|=0$. Решение этого уравнения имеет вид $\hat{\phi}_{1}=\widehat{Q} \hat{\phi} \star \hat{\phi}$. Продолжая дальше итерации, мы получим $\hat{\phi}_{n}=\widehat{Q} \hat{\phi} \star \hat{\phi}^{n}$, и тогда $\widehat{\Phi}$ имеет вид

$$
\widehat{\Phi}=\sum_{n=0}^{\infty} \lambda^{n+1} \widehat{Q} \hat{\phi} \star \hat{\phi}^{n}=\lambda \widehat{Q} \hat{\phi} \frac{1}{1-\lambda \hat{\phi}}
$$

Это выражение можно переписать как

$$
\widehat{\Phi}=\left(Q \phi_{+} \otimes \sigma_{3}+Q \phi_{-} \otimes i \sigma_{2}\right) \frac{1}{\left(1-\lambda \phi_{+}\right)^{2}-\lambda^{2} \phi_{-}^{2}}\left(\left(1-\lambda \phi_{+}\right) \otimes I+\lambda \phi_{-} \otimes \sigma_{1}\right) .
$$

Отсюда получаем, что

$$
\begin{aligned}
& \Phi_{+}=\frac{\lambda}{2} Q\left(\phi_{+}+\phi_{-}\right) \frac{1}{1-\lambda\left(\phi_{+}+\phi_{-}\right)}+\frac{\lambda}{2} Q\left(\phi_{+}-\phi_{-}\right) \frac{1}{1-\lambda\left(\phi_{+}-\phi_{-}\right)}, \\
& \Phi_{-}=\frac{\lambda}{2} Q\left(\phi_{+}+\phi_{-}\right) \frac{1}{1-\lambda\left(\phi_{+}+\phi_{-}\right)}-\frac{\lambda}{2} Q\left(\phi_{+}-\phi_{-}\right) \frac{1}{1-\lambda\left(\phi_{+}-\phi_{-}\right)},
\end{aligned}
$$

что совпадает с полученным ранее результатом [9].

\section{4. ЭКВИВАЛЕНТНОСТЬ КУБИЧЕСКОЙ И НЕПОЛИНОМИАЛЬНЫХ NS-ТЕОРИЙ C УЧЕTOM GSO(-)-CEKTOPA}

4.1. Эквивалентность теорий в $\mathrm{GSO}(+)$-секторе. Напомним основные идеи и формулы эквивалентности классических решений кубической [2], [3] и неполиномиальной [12] теорий в $\mathrm{GSO}(+)$-секторе, полученные в работе [13] (мы перечислим основные факты, а детали будут продемонстрированы в следующем разделе после включения $\mathrm{GSO}(-)$-сектора).

Напомним, что уравнение движения струнного поля $G$ в неполиномиальной теории записывается как

$$
\eta_{0}\left(G^{-1} Q G\right)=0 .
$$

Здесь, в отличие от кубической полевой теории фермионной NS-струны, поле $G$ является элементом расширенной алгебры [14], при этом соотношение (24) означает, что $G^{-1} Q G$ лежит в малой алгебре ${ }^{1)}$.

Определим поле $\Psi$, соответствующее $G$, формулой [13]

$$
\Psi=G^{-1} Q G .
$$

Это поле является чистой калибровкой с той оговоркой, что $G$ принадлежит большой алгебре, и поэтому $\Psi$ удовлетворяет уравнению движения (1). Однако если $G$ удовлетворяет уравнению движения неполиномиальной теории (24), то поле (25) лежит в малой алгебре и, следовательно, является решением кубической теории. Таким образом определено отображение $g$ пространства решений неполиномиальной

\footnotetext{
1) Здесь и ниже слова "большая" и "малая" алгебра мы употребляем в том смысле, в каком они употребляются в работе [14].
} 


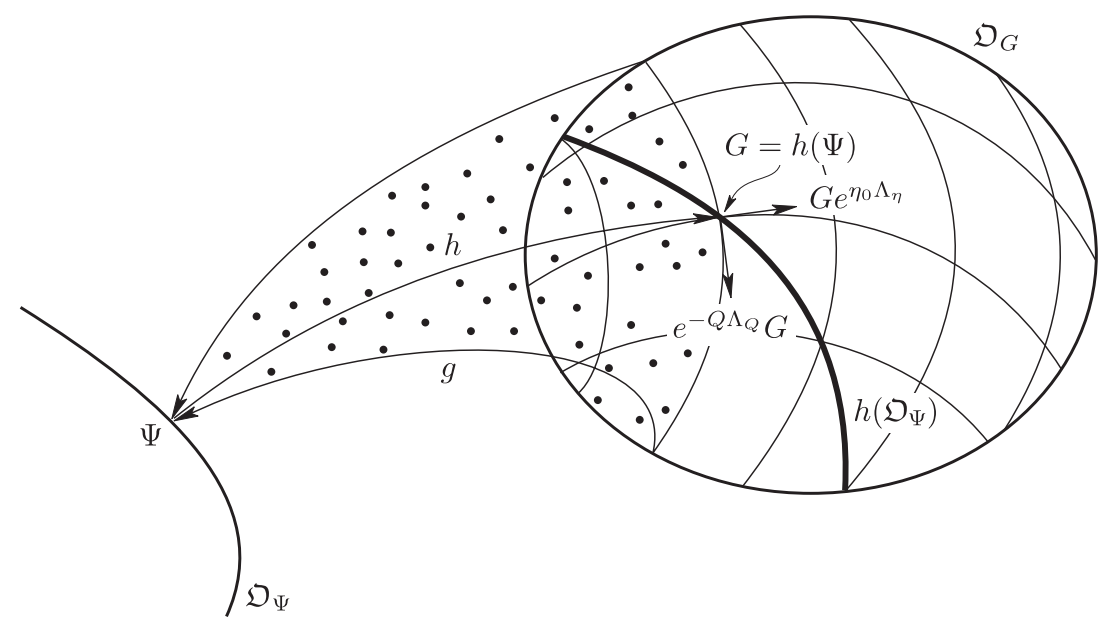

Отображения $h$ и $g$.

теории (обозначим это пространство через $\mathfrak{B}$ ) в пространство решений кубической теории (обозначим его через $\mathfrak{A})$ (см. рисунок):

$$
g: \mathfrak{B} \rightarrow \mathfrak{A}, \quad g(G)=\Psi, \quad \Psi=G^{-1} Q G, \quad G \in \mathfrak{B} .
$$

Далее определим оператор $P$, включающий в себя оператор смены картины $Y(z)$ и поле $\xi(z)$, а именно

$$
P=\frac{e^{i \pi / 4} P(i)+e^{-i \pi / 4} P(-i)}{\sqrt{2}}, \quad P(z)=(\xi Y)(z)=-\left(c \xi \partial \xi e^{-2 \phi}\right)(z)
$$

где $c, \xi$ и $\phi$ - духовые поля (мы, как и в работе [13], используем обозначения работы [4]). Важными свойствами такого оператора являются (см. работу [13]) равенство

$$
\left(P \Psi_{1}\right) \star\left(P \Psi_{2}\right)=0
$$

и правило перестановки с BRST-зарядом

$$
\{Q, P(z)\}=1 \text {. }
$$

Оператор $P$ позволяет определить отображение $h: \mathfrak{A} \rightarrow \mathfrak{B}$ в соответствии с формулой $h: \Psi \rightarrow G=e^{P \Psi}$ (см. рисунок). Если $\Psi$ удовлетворяет уравнению (1), то $G=h(\Psi)$ удовлетворяет уравнению $(24)$, иными словами, $h(\mathfrak{A}) \subset \mathfrak{B}$. Доказательство этого факта сводится к проверке того, что $G^{-1} Q G$ для поля $G=h(\Psi)$ лежит в малой алгебре, и основано на свойствах оператора $P$ и на том, что $\Psi$ есть решение в кубической теории.

Отображения $h$ и $g$ нетривиально связаны. Рассмотрим сначала композицию $g \circ h$. Для любого $\Psi \in \mathfrak{A}$ имеем $(g \circ h)(\Psi)=e^{-P \Psi} Q e^{P \Psi}=\Psi$. Следовательно, $g \circ h=\mathrm{Id}$ и $g(\mathfrak{B})=\mathfrak{A}$, т.е. любое классическое решение в кубической теории представимо 
в виде "чистой калибровки". Рассмотрим теперь композицию $h \circ g$. В работе [13] доказано, что $(h \circ g)(G) \neq G$, однако

$$
(h \circ g)(G)=e^{-Q P\left(1-G^{-1}\right)} G,
$$

т.е. $(h \circ g)(G)$ принадлежит калибровочной орбите $\mathfrak{O}_{G}=\left\{\widetilde{G}: \widetilde{G}=e^{-Q \Lambda_{Q}} G e^{\eta_{0} \Lambda_{\eta}}\right\}$ исходного поля $G$. Именно в терминах калибровочных орбит может быть получено наиболее прозрачное описание отображений $g$ и $h$.

Пусть $\Psi$ - произвольное поле из $\mathfrak{A}$ и $G=h(\Psi)$. Рассмотрим образ орбиты $\mathfrak{O}_{\Psi}=$ $\left\{\widetilde{\Psi}: \widetilde{\Psi}=e^{-\Lambda}(\Psi+Q) e^{\Lambda}\right\}$ при отображении $h: h\left(\mathfrak{O}_{\Psi}\right)=\{\widetilde{G}: \widetilde{G}=h(\widetilde{\Psi})\}$. Прямое вычисление дает [13]

$$
\begin{aligned}
\widetilde{G} & =1+P \widetilde{\Psi}=1+P\left(e^{-\Lambda}(\Psi+Q) e^{\Lambda}\right)=1+P\left(-Q e^{-\Lambda}+e^{-\Lambda} \Psi\right) e^{\Lambda}= \\
& =\left(Q\left(P e^{-\Lambda}\right)+P e^{-\Lambda} \Psi\right) e^{\Lambda}=Q\left(P e^{-\Lambda}\right)(1+P \Psi) e^{\Lambda}=Q\left(P e^{-Q P \Lambda}\right) G e^{\Lambda}= \\
& =e^{-Q P \Lambda} G e^{\Lambda}=e^{-Q P \Lambda} G e^{\eta_{0} \xi \Lambda}
\end{aligned}
$$

т.е. $h\left(\mathfrak{O}_{\Psi}\right)$ есть подорбита поля $G=h(\Psi)$, заданная специальным выбором калибровочных параметров $\Lambda_{Q}, \Lambda_{\eta}$ или отображения $h\left(\mathfrak{O}_{\Psi}\right) \subset \mathfrak{O}_{G}$.

Пусть теперь $G$ - произвольное поле из $\mathfrak{B}$ и $\Psi=g(G)$. Рассмотрим образ орбиты $\mathfrak{O}_{G}$ при отображении $g$, т.е. $g\left(\mathfrak{O}_{G}\right)=\left\{\widetilde{\Psi}=g(\widetilde{G}): \widetilde{G} \in \mathfrak{O}_{G}\right\}$. Имеем

$$
\begin{aligned}
\widetilde{\Psi} & =\widetilde{G}^{-1} Q \widetilde{G}=e^{-\eta_{0} \Lambda_{\eta}} G^{-1} e^{Q \Lambda_{Q}} Q\left(e^{-Q \Lambda_{Q}} G e^{\eta_{0} \Lambda_{\eta}}\right)= \\
& =e^{-\eta_{0} \Lambda_{\eta}} G^{-1}\left((Q G) e^{\eta_{0} \Lambda_{\eta}}+G Q e^{\eta_{0} \Lambda_{\eta}}\right)=e^{-\eta_{0} \Lambda_{\eta}}(\Psi+Q) e^{\eta_{0} \Lambda_{\eta}} .
\end{aligned}
$$

В силу произвольности $\Lambda_{\eta}$ получаем, что $g\left(\mathfrak{O}_{G}\right)=\mathfrak{O}_{\Psi}$. Заметим теперь, что если $h\left(\Psi^{\prime}\right) \in \mathfrak{O}_{h(\Psi)}$, то $\Psi^{\prime} \in \mathfrak{O}_{\Psi}$. Действительно, в силу $g \circ h=\operatorname{Id}$ можно написать $\Psi^{\prime}=g\left(h\left(\Psi^{\prime}\right)\right)$, и, поскольку $g\left(\mathfrak{O}_{G}\right)=\mathfrak{O}_{\Psi}$, то $h\left(\Psi^{\prime}\right) \in \mathfrak{O}_{\Psi}$.

Таким образом, мы видим, что отображения $g$ и $h$ могут быть сужены до отображений $h: \mathfrak{O}_{\Psi} \rightarrow \mathfrak{O}_{G}, g: \mathfrak{O}_{G} \rightarrow \mathfrak{O}_{\Psi}$ соответствующих орбит. При этом образ $\mathfrak{O}_{\Psi}$ в $\mathfrak{O}_{G}$ есть подорбита, задаваемая формулой (30). Образом $\mathfrak{O}_{G}$ служит вся орбита $\mathfrak{O}_{\Psi}$, при этом все элементы $\mathfrak{O}_{G}$, отличающиеся только значением $\Lambda_{Q}$, отображаются в один и тот же элемент $\mathfrak{O}_{\Psi}$ (см. (31)). Суженное на $h\left(\mathfrak{O}_{\Psi}\right)$ отображение $g$ становится обратимым: $\left.h \circ g\right|_{h\left(\mathcal{O}_{\Psi}\right)}=\mathrm{Id}$. Композиция $h \circ g$ задает в орбите $\mathfrak{O}_{G}$ специальное сечение (29).

Таким образом установлено взаимно однозначное (с точностью до калибровки) соответствие между решениями двух теорий в $\mathrm{GSO}(+)$-секторах.

\section{2. Эквивалентность кубической и неполиномиальной теорий с учетом} GSO(-)-сектора. В этом пункте мы переносим схему рассуждений из предыдущего пункта на теорию, включающую GSO(-)-сектор, используя матричное описание.

Формулировка кубической теории NS-струны, включающей GSO(-)-сектор, приведена в разделе 1 . В неполиномиальной теории $\widehat{G}=G_{+} \otimes I+G_{-} \otimes \sigma_{1}$, и уравнения движения сохраняют прежний вид $(24)$ с $\hat{\eta}_{0} \equiv \eta \otimes \sigma_{3}$. Из условия $\widehat{G}^{-1} \widehat{G}=I$ следует, что $G_{+} G_{-}=G_{-} G_{+}$. 
Пусть теперь $\mathfrak{A}$ есть множество матричных решений уравнения движения (8) и $\mathfrak{B}$ есть множество решений уравнения (24). Определим отображение $g$ множества $\mathfrak{B}$ на множество $\mathfrak{A}$ формулой $\widehat{\Psi} \equiv g(\widehat{G})=\widehat{G}^{-1} \widehat{Q} \widehat{G}$. Покомпонентно последнее соотношение может быть переписано как

$$
\Psi_{+}=G_{+}^{-1} Q G_{+}+\frac{G_{-}}{G_{+}^{2}} Q G_{-}, \quad \Psi_{-}=G_{+}^{-1} Q G_{-}+\frac{G_{-}}{G_{+}^{2}} Q G_{+} .
$$

Для того чтобы $\widehat{\Psi}=g(\widehat{G})$ являлось решением уравнения движения (8), необходимо и достаточно, чтобы для оператора $\widehat{Q}$ было выполнено правило Лейбница, аналогичное (7). Это правило действительно выполнено, поскольку поле $G_{+}$выбирается четным, а поле $G_{-}$- нечетным.

Отображение множества $\mathfrak{A}$ в множество $\mathfrak{B}$ зададим формулой

$$
\widehat{G}=h(\widehat{\Psi})=e^{\widehat{P} \widehat{\Psi}}=1+\widehat{P} \widehat{\Psi}
$$

(здесь под 1 мы понимаем единичное относительно $\star$ состояние $|I\rangle \otimes I$, а $\widehat{P} \equiv P \otimes \sigma_{3}$ ), и это обеспечит справедливость соотношений $(27)$ и (28) для $\widehat{P}$.

В компонентах отображение (33) записывается как

$$
G_{+}=1+P \Psi_{+}=e^{P \Psi_{+}}, \quad G_{-}=P \Psi_{-},
$$

при этом

$$
\widehat{G}^{-1}=\left(1-P \Psi_{+}\right) \otimes I-P \Psi_{-} \otimes \sigma_{1}=G_{+}^{-1} \otimes I-G_{-} \otimes \sigma_{1}=e^{-\widehat{P} \widehat{\Psi}} .
$$

Рассмотрим композицию $g \circ h$. Для $\widetilde{\Psi}=(g \circ h)(\widehat{\Psi})$ имеем

$$
\begin{aligned}
\widetilde{\Psi} & =(g \circ h)(\widehat{\Psi})=(1-\widehat{P} \widehat{\Psi}) \widehat{Q}(1+\widehat{P} \widehat{\Psi})=(1-\widehat{P} \widehat{\Psi}) \widehat{Q} \widehat{P} \widehat{\Psi}=(1-\widehat{P} \widehat{\Psi})(1-\widehat{P} \widehat{Q}) \widehat{\Psi}= \\
& =(1-\widehat{P} \widehat{Q}-\widehat{P} \widehat{\Psi}) \widehat{\Psi}=\widehat{\Psi}-\widehat{P}\left(\widehat{Q} \widehat{\Psi}+\widehat{\Psi}^{2}\right)=\widehat{\Psi}
\end{aligned}
$$

здесь мы использовали тот факт, что $\widehat{\Psi}$ по определению удовлетворяет уравнению движения, а также матричные аналоги формул (27), (28). Таким образом, мы доказали, что $f \circ g=\mathrm{Id}$.

Рассмотрим теперь композицию $h \circ g$. Для $\widetilde{\widehat{G}}=h \circ g(\widehat{G})$ имеем

$$
\begin{aligned}
\widetilde{\widehat{G}} & =h \circ g(\widehat{G})=e^{\widehat{P} \widehat{G}^{-1} \widehat{Q} \widehat{G}}=1+\widehat{P} \widehat{G}^{-1} \widehat{Q} \widehat{G}=1-\widehat{P} \widehat{Q} \widehat{G}^{-1} \cdot \widehat{G}= \\
& =1-(1-\widehat{Q} \widehat{P}) \widehat{G}^{-1} \cdot \widehat{G}=1-1+\widehat{Q} \widehat{P} \widehat{G}^{-1} \cdot \widehat{G}=\widehat{Q} \widehat{P} \widehat{G}^{-1} \cdot \widehat{G} .
\end{aligned}
$$

Введем следующую параметризацию [13] для произвольного $\widehat{G} \in \mathfrak{B}:$

$$
\widehat{G}=\frac{1}{1-\hat{\phi}} .
$$


Элемент $\widehat{\Psi}=g(\widehat{G}) \in \mathfrak{A}$, построенный из $\widehat{G}$, при такой параметризации примет вид $(\widehat{G}-$ четный элемент)

$$
\widehat{\Psi}=\widehat{G}^{-1} \widehat{Q} \widehat{G}=-\widehat{Q} \widehat{G}^{-1} \widehat{G}=\widehat{Q} \hat{\phi} \frac{1}{1-\hat{\phi}} .
$$

Здесь мы использовали правило Лейбница, при этом для нас было важно, что четности $G_{+}$и $G_{-}$различны, а также, что $\sigma_{3} I=I \sigma_{3}, \sigma_{3} \sigma_{1}=-\sigma_{1} \sigma_{3}$, и то, что оператор $P$ меняет четность поля на противоположную.

Воспользуемся параметризацией (36) для (35), тогда

$$
\widetilde{\widehat{G}}=\widehat{Q} \widehat{P} \widehat{G}^{-1} \widehat{G}=\widehat{Q} \widehat{P}(1-\hat{\phi}) \frac{1}{1-\hat{\phi}}=\frac{1}{1-\hat{\phi}}-\widehat{Q} \widehat{P} \hat{\phi} \frac{1}{1-\hat{\phi}}=(1-\widehat{Q}(\widehat{P} \hat{\phi})) \widehat{G},
$$

где мы учли, что $\widehat{Q} \widehat{P} I=I$. Перепишем формулу (37) в виде

$$
\widetilde{\widehat{G}}=e^{-\widehat{Q}(\widehat{P} \hat{\phi})} \widehat{G},
$$

что соответствует калибровочному преобразованию с $\widehat{\Lambda}_{Q}=\widehat{P} \hat{\phi}, \widetilde{G}=e^{-\widehat{Q} \widehat{\Lambda}_{Q}} \widehat{G}^{\widehat{\eta}_{0} \widehat{\Lambda}_{\eta}}$. Таким образом мы установили, что отображение $h \circ g$ отображает элемент $\widehat{G}$ в его калибровочную орбиту. В компонентах преобразование (38) запишется в виде

$$
\widetilde{G}_{+}=e^{-Q \Lambda_{+}} G_{+}-Q \Lambda_{-} G_{-}, \quad \widetilde{G}_{-}=e^{-Q \Lambda_{+}} G_{-}-Q \Lambda_{-} G_{+},
$$

где $\Lambda_{+}=P \Phi_{+}, 0 \Lambda_{-}=P \Phi_{-}$.

\section{5. ЗАКЛЮЧЕНИЕ}

В настоящей статье мы построили матричное представление нулевой кривизны для струны, включающей $\mathrm{GSO}(+)$ - и $\mathrm{GSO}(-)$-секторы. С помощью этого представления доказана эквивалентность подходов, использующих кубическое и неполиномиальное действие.

Благодарности. Работа частично поддержана РФФИ (грант № 08-01-00798) и Программой поддержки ведущих научных школ (грант НШ-795.2008.1). Работа И. Я. Арефьевой частично поддержана INTAS (грант № 03-51-6346).

\section{Список литературы}

[1] E. Witten, Nucl. Phys. B, 276:2 (1986), 291-324.

[2] I. Ya. Aref'eva, P. B. Medvedev, A. P. Zubarev, Nucl. Phys. B, 341:2 (1990), 464-498.

[3] C. R. Preitschopf, C. B. Thorn, S. A. Yost, Nucl. Phys. B, 337:2 (1990), 363-433.

[4] I. Ya. Aref'eva, D. M. Belov, A. A. Giryavets, A. S. Koshelev, P. B. Medvedev, "Noncommutative field theories and (super)string field theories", Particles and Fields (São Paulo, Brazil 14-27 January 2001), eds. G. A. Alves, O. J.P. Eboli, V. O. Rivelles, World Sci., River Edge, NJ, 2002, 1-163; arXiv: hep-th/0111208.

[5] M. Schnabl, Adv. Theor. Math. Phys., 10:4 (2006), 433-501; arXiv: hep-th/0511286.

[6] Y. Okawa, JHEP, 04 (2006), 055; arXiv: hep-th/0603159. 
[7] E. Fuchs, M. Kroyter, JHEP, 05 (2006), 006; arXiv: hep-th/0603195; T. Erler, JHEP, 05 (2007), 083; 084; arXiv: hep-th/0611200; arXiv: hep-th/0612050; T. Takahashi, JHEP, 01 (2008), 001; arXiv: hep-th/0710.5358.

[8] I. Ya. Aref'eva, D. M. Belov, A. S. Koshelev, P. B. Medvedev, Nucl. Phys. B, 638:1-2 (2002), 21-40; arXiv: hep-th/0107197.

[9] I. Ya. Aref'eva, R. V. Gorbachev, P. B. Medvedev, Tachyon Solution in Cubic Neveu-Schwarz String Field Theory, arXiv: 0804.2017.

[10] I. Ya. Aref'eva, D. M. Belov, A. A. Giryavets, JHEP, 09 (2002), 050; arXiv: hep-th/0201197.

[11] N. Berkovits, A. Sen, B. Zwiebach, Nucl. Phys. B, 587:1-3 (2000), 147-178; arXiv: hep-th/0002211.

[12] N. Berkovits, Nucl. Phys. B, 450:1-2 (1995), 90-102; Erratum, 459:1-2, 439-451; arXiv: hep-th/9503099.

[13] E. Fuchs, M. Kroyter, JHEP, 10 (2008), 054; arXiv: hep-th/0805.4386.

[14] D. Friedan, E. Martinec, S. Shenker, Nucl. Phys. B, 271:1 (1986), 93-165.

Поступила в редакцию 16.09.2008 